\title{
BMJ Open Longitudinal Youth in Transition Study (LYiTS): protocol for a multicentre prospective cohort study of youth transitioning out of child and adolescent mental health services at age 18
}

Kristin Cleverley (10 ,1,2,3 Kathryn J Bennett, ${ }^{4}$ Sarah Brennenstuhl, ${ }^{1}$ Amy Cheung, ${ }^{3,5}$ Joanna Henderson (D) ,2,3 Daphne J Korczak, ${ }^{3,6}$ Paul Kurdyak, ${ }^{2,3}$ Andrea Levinson,, ${ }^{2,3}$ Antonio Pignatiello, ${ }^{3,6}$ Jennifer Stinson, ${ }^{1,7}$ Aristotle N Voineskos, ${ }^{2,3}$ Peter Szatmari ${ }^{2,3,6}$

To cite: Cleverley K, Bennett KJ, Brennenstuhl S, et al. Longitudinal Youth in Transition Study (LYiTS): protocol for a multicentre prospective cohort study of youth transitioning out of child and adolescent mental health services at age 18. BMJ Open 2020;10:e035744. doi:10.1136/ bmjopen-2019-035744

- Prepublication history for this paper is available online. To view these files, please visit the journal online (http://dx.doi. org/10.1136/bmjopen-2019035744).

Received 13 November 2019 Revised 14 January 2020 Accepted 15 January 2020
Check for updates

(c) Author(s) (or their employer(s)) 2020. Re-use permitted under CC BY-NC. No commercial re-use. See rights and permissions. Published by BMJ.

For numbered affiliations see end of article.

Correspondence to Dr Kristin Cleverley; k.cleverley@utoronto.ca

\section{ABSTRACT}

Introduction Transition between health services is widely recognised as a problematic hurdle. Yet, the factors necessary for successful transition out of child and adolescent mental health services (CAMHS) as youth reach the service boundary at age 18 are poorly understood. Further, fragmentation and variability among the services provided by mental health organisations serve to exacerbate mental illness and create unnecessary challenges for youth and their families. The primary aim of the Longitudinal Youth in Transition Study (LYiTS) is to describe and model changes in psychiatric symptoms, functioning and health service utilisation at the transition out of CAMHS at age 18 and to identify key elements of the transition process that are amendable to interventions aimed at ensuring continuity of care.

Methods and analysis A prospective longitudinal cohort study will be conducted to examine the association between psychiatric symptoms, functioning and mental health and health service use of youth aged 16-18 as they transition out of child mental health services at age 18. We will recruit a sample of $(n=350)$ participants from child and adolescent psychiatric programmes at two hospital and two community mental health sites and conduct assessments annually for 3 years using standardised measures of psychiatric symptoms, functioning and health service utilisation.

Ethics and dissemination Ethics approval has been obtained at all four recruitment sites. We will disseminate the results through conferences, open access publications and webinars.

\section{INTRODUCTION}

A high percentage of child-onset psychiatric disorders persist into adulthood ${ }^{1}$; thus, the need to transition from child to adult care is a reality for many youth. Yet the transition from child and adolescent mental health services (CAMHS) to adult mental health services (AMHS) is typically fragmented ${ }^{2}$ and poorly

\section{Strengths and limitations of this study}

- Longitudinal Youth in Transition Study (LYiTS) is the first prospective longitudinal cohort study conducted to track youths' experiences of transition in mental healthcare at age 18 in North America.

- LYiTS will link self-reported private and public mental health service utilisation data with a publicly funded administrative health services records database.

- Youth will be recruited from both community and hospital sites.

- While very detailed subject contact management plans have been developed with youth input, it is likely that there will still be high rates of attrition.

- This study is restricted to participants who can read/ write in English.

coordinated. ${ }^{34}$ Research from the US and UK has shown that mental health service utilisation at the transition to adulthood declined up to $50 \%-60 \%{ }^{56}$ As such, there is a need to identify youth who are most likely to experience poor continuity in mental healthcare transitions, resulting in negative health and social outcomes, and who may benefit from transition interventions. A recent systematic review on healthcare transition service models for child to adult physical healthcare services found that there was limited evidence to support the creation of standards of care or clinical guidelines for transitions. ${ }^{7}$ This reflects the paucity of longitudinal data on transitions in care, transition planning and post-transfer outcomes. ${ }^{89}$ Studies are urgently needed to empirically derive individual and program-level risk factors for poor outcomes that are amenable to interventions. ${ }^{10}$ 
In mental healthcare, effective transitions aim to ensure continuity of care through a planned healthcare process that addresses both the therapeutic and developmental needs of the youth. ${ }^{11}$ The current hypothesis is that successful transitions from CAMHS to AMHS is contingent on the youth experiencing continuity of care and avoiding decrements in their mental health and functioning. Experiencing discontinuity in mental healthcare at age 18 has been associated with developing more severe and enduring mental health problems, increased frequency of risky behaviours, inadequate/improper medication monitoring, increased involvement with the justice system and decreased social support from caregivers. ${ }^{6}{ }^{12-15}$ As such, to inform service delivery models and clinical interventions, clinicians and researchers have begun to propose criteria to assess continuity of care and evaluate the success of transitions from CAMHS to AMHS. One study, the transition from CAMHS to AMHS (TRACK) project retrospectively evaluated four elements of successful transitions (gradual preparation, transfer planning, periods of parallel care and consistency of key worker ${ }^{616}$ ) in the medical records of 154 youth. ${ }^{6}$ Most youth $(78 \%)$ experienced suboptimal transitions (1-3 of these criteria), less than $5 \%$ experienced all 4 and $17 \%$ experienced none.

The TRACK study has been highly influential in reshaping the practices and policies of mental health transitions, including the development of National Institute for Health and Care Excellence guidelines in the UK. ${ }^{17}$ Stakeholders, including youth and their caregivers, clinicians and administrators, are demanding similar practice and policy reform in other parts of the world, including Canada. ${ }^{3} 1819$ However, to date, there has been no published prospective longitudinal studies to inform policy-makers on the number of youth with mental illness who experience a disruptive transition in care from CAMHS at age 18, and the impact that a disruptive transition has on their mental health and functioning. These data are essential in strategic service planning of youth reaching 18 years. Our prospective longitudinal study will generate new knowledge that can address these gaps.

\section{Study aims}

The primary aim of the Longitudinal Study of Child and Youth (LYiTS) is to document the mental health and functioning trajectories of youth aged 16-18 receiving CAMHS as they cross the CAMHS/AHMS transition boundary. The secondary aims are to: (1) examine whether specific personal and service prognostic factors are associated with varied mental health and functioning trajectories; and (2) characterise the rates of mental health and primary care service use 1 year before and 1 year after transitioning out of CAMHS.

\section{METHODS AND ANALYSIS}

\section{Study design}

LYiTS is a prospective longitudinal cohort study of youth age 16-18 years who currently receive CAMHS and reach the CAMHS/AMHS transition boundary. Postbaseline follow-up data will be collected annually for 3 years [time 1 (baseline), time 2 (12 months), time 3 (24 months), time 4 (36 months)] on a clinically referred sample of youth receiving outpatient treatment in one of four CAMHS clinics. The age range of $16-18$ years was chosen to ensure that data collection occurred over the transition boundary of age 18 years. Four time points were chosen to be long enough to reflect change in mental health, functioning and experiences in transitions in mental healthcare.

\section{Study setting}

Participants will be recruited from the Child, Youth and Family Program at the Centre for Addiction and Mental Health (CAMH), the Department of Psychiatry at the Hospital for Sick Children ('SickKids'), the SickKids Centre for Community Mental Health (CCMH; formally known as the Hincks Dellcrest Centre) and The George Hull Centre for Children and Families (GHC), all in Toronto, Ontario, Canada. CAMH and SickKids are large tertiary hospitals that provide inpatient and outpatient services to youth with mental health problems, singleentry intakes and research registries that will facilitate participant recruitment. CCMH and GHC are community mental health agencies with a structured intake team providing outpatient services, day treatment services and adolescent residential programme. All sites serve adolescents, have an age 18 discharge requirement and are located in Toronto, Ontario, Canada.

\section{Study population}

\section{Inclusion criteria}

Eligible participants are those who are 16 to 18 years old and are currently receiving outpatient treatment in CAMHS. All participants must be able to speak and read English. To be eligible, currently receiving services was defined as a youth having an appointment with a CAMHS clinician at least once, not including the initial consult/ assessment, within the last 6 months.

\section{Exclusion criteria}

The exclusion criteria include any youth who are enrolled in a single service that extends beyond age 18 (eg, early intervention psychosis programme) as these youth would experience different transitions in care. Also, any youth who is currently experiencing distress (eg, psychosis or acuity requiring immediate hospitalisation) will be excluded.

\section{Sampling, recruitment and consent}

A comprehensive recruitment and contact management plan was developed in collaboration with stakeholders (youth, caregivers) and direct service clinicians. This recruitment plan was tested in the feasibility pilot and minor revisions were made. Consecutive eligible clients of CAMHS will be recruited for approximately 36 months starting from the time of Research Ethics Board (REB) approval at each site. We will recruit 350 youth aged 16-18 years across the four study sites proportional to the 
eligible sample at the site. This age will ensure youth will cross the service age boundary of 18 years.

At each recruitment site, youth are screened based on the eligibility criteria and deemed either eligible or ineligible to enrol. All youth who have a scheduled appointment during the recruitment period will be screened for eligibility. Once a youth is screened as eligible, a clinician from the circle of care (ie, physician, nurse, social worker, psychologist) approaches the youth, provides them with a participation information letter about the study, and seeks agreement from the youth for a research assistant (RA) to meet with them to introduce the study. If the youth is agreeable, the RA meets with the youth to provide study information and answer questions. If, however, a youth prefers to discuss the project at a later time, they will be given a study information sheet and the RA will follow-up via their preferred method of contact. Once the youth is agreeable to participate, consent is obtained, and the RA sets up a time to conduct the initial study visit. Given that the study is recruiting youth with identified mental health problems, who may not be receiving ongoing mental healthcare after the baseline assessment, a clinical backup is always available to the RA, and youth will be given a handout on local service options should they need them. Recruitment for the feasibility pilot study occurred from June 2016 to February 2017 at CAMH and CCMH. Recruitment for the study presented here commenced on revised ethics board approval on February 2017 at CAMH and CCMH, and ethics approval on January 2018 at GHC, and September 2018 at Sickkids. Baseline recruitment is scheduled to conclude in July 2020. It is anticipated the final participant will complete all assessments in July 2023.

\section{Data collection}

Participants complete self-report study measures annually for 3 years following baseline, comprising four time points (T1-T4). The study measures take approximately 45-90 min to complete. A study RA remains with the participant during the completion of the self-report measures to address questions or concerns.

\section{Measures}

Participants complete all measures at all four time points, except the Youth Transition Questionnaire (YTQ) and the Continuity of Care in Children's Mental HealthYouth Version (C3MH-Y). The YTQ assesses pretransition preparation and planning in CAMHS and the C3MH-Y measures relationships with CAMHS clinicians. As such, both measures are only completed until the time point immediately following discharge from CAMHS.

\section{Outcome measures}

\section{Mental health and functioning}

Youth will complete the Youth Self Report (YSR), ${ }^{20}$ a 119-item scale which measures psychiatric symptoms and adaptive functioning. Items are rated as 0 (not true), 1 (somewhat or sometimes true) or 2 (very true or often true). The YSR yields dimensional 'total problem scores' of symptoms corresponding to the six most common Diagnostic and Statistical Manual (DSM) diagnoses among youth. Once youth turn 19, they will then complete the adult version (Adult Self Report (ASR) ${ }^{21}$ ) of the YSR. Structured in the same way as the YSR, the ASR has 126 questions and assesses psychiatric symptoms and adaptive functioning. Mental health will also be assessed using three other measures to ensure a comprehensive assessment of mental health. The Strengths and Difficulties Questionnaire is a 25-item instrument comprising five scales (emotional problems, conduct problems, hyperactivity, peer problems and prosocial). Items are scored from 0 (not true), 1 (somewhat true) and 3 (certainly true). A total difficulties score is generated by summing scores from all scales except the prosocial scale, with a total possible score of 40 and higher scores indicting more problems. ${ }^{22}{ }^{23}$ The Difficulty in Emotional Regulation Scale is a 36-item instrument that will be used to assess six aspects (non-acceptance, goals, impulse, awareness, strategies and clarity) of emotion dysregulation. ${ }^{24}$ Each of the items is scored using a 5-point scale (ranging from $1=$ almost never to $5=$ almost always), total scores range from 36 to 180 with greater scores indicating more emotion dysregulation. The CAGE-Adapted to Include Drugs questionnaire will be used to identify high-risk alcohol disorders and other drug use problem behaviours. ${ }^{25}$ This 4 -item questionnaire is scored using 0 (no) and 1 (yes) with scores added for a possible total score of 4 . A total score of 2 or greater is considered clinically significant. Functional impairment will be assessed using the Columbia Impairment Scale, ${ }^{26}$ a 13-item scale which provides a global measure of impairment in 4 major areas of functioning: interpersonal relations (family and friends), broad psychopathological domains, functioning in job or schoolwork and use of leisure time. Items are scored from 0 (no problem) to 4 (a very big problem), with higher total scores indicating poorer functioning.

\section{Predictors}

Personal characteristics

To describe the basic characteristics of the study sample, youth will complete a basic demographic form. Personal characteristics such as social support, developmental maturity and transition readiness will be measured at each time point. The Transition Readiness Assessment Questionnaire (TRAQ) ${ }^{27}$ measures youth's readiness (related to skills, knowledge and self-efficacy) to transition from child-oriented to adult-oriented mental health services. The TRAQ is a 20 -item instrument, scored on a 5-point Likert scale. Total scores $(/ 100)$ will be generated, with higher scores indicating greater readiness and ability to negotiate the adult healthcare system. ${ }^{27}{ }^{28}$ Perceived social support will be assessed using the Multidimensional Scale of Perceived Social Support (MSPSS) ${ }^{29}$ The MSPSS is a 12-item instrument, measuring perception of social support with family, friends and a significant other, measured on a 7-point Likert scale. A total score (/84) and domain-specific scores $(/ 28)$ are calculated with 
higher scores indicating greater perceived social support. Self-perceived maturity for handling tasks of emerging adulthood (ages 18-25 years) will be measured using the Inventory of the Dimensions of Emerging Adulthood ${ }^{30}$ a 31-item instrument measured on a 4-point Likert scale. Total scores will be generated, with higher scores indicating greater developmental maturity.

\section{Service factors}

Youth will complete the Health and Social Service Utilization Measure, ${ }^{31}$ a structured interview that assesses use of health services that are billable (physician visits, emergency room visits) and non-billable (private therapist, community drop-in agencies, online/telephone counselling, school counsellor, etc.) to the government-run health insurance plan. Linkage to health insurance data (via the Ontario Health Insurance Plan, OHIP) will provide information about billable visits, including physician, emergency room and ambulatory and hospital visits. Administrative healthcare data (OHIP), such as healthcare usage and costs (ie, physician billings, hospital stays and emergency use), is housed at the Institute for Clinical Evaluative Sciences (ICES). Composite measures of health service utilisation will be generated: (1) the total number of visits (all providers) in the 1-year pre-CAMHS and 1-year post-CAMHS discharge; and (2) the total number of unscheduled (emergent) visits to the ER, walk-in clinic use, and telephone/internet crisis line use in the 1-year pre-CAMHS and 1-year post-CAMHS discharge. Two other service-level factors will be reported at each time point, the length of service use and organisation type. The length of service use with current mental health programme/organisation will be reported by youth and verified via OHIP in total months. The four recruitment sites represent different types of healthcare organisations and will be coded as a categorical nominal variable. CAMH is a psychiatric hospital with CAMHS and AMHS; SickKids is a paediatric hospital with only CAMHS; and GHC and CCMH are both community paediatric mental health organisation with only CAMHS.

\section{Program factors}

Detailed descriptions of transition plans, preparation and youth's experience of continuity of care during the transition period will be assessed at each time point during the study. Transition plan and preparation experienced by the youth will be assessed using the YTQ, composed of six items assessed in the TRACK study. ${ }^{32}$ The YTQ will be completed at each time point prior to transitioning out of CAMHS. YTQ items, scored using yes or no responses (with open-text comment boxes to expand), include: (1) Has a transition or referral to AMHS been discussed with you at any of your recent meetings with your clinician/ clinical team? (2) Has a referral to AMHS been sent? (3) Was the reason for moving to AMHS discussed with you? (4) Were you provided with information about AMHS? (5) Were you involved in the discussion and decisionmaking about when you would move to AMHS and where you would be going? (6) Has your family (eg, parents, siblings or partner) been involved in the discussion about moving to AMHS?

Youth will complete the C3MH-Y ${ }^{33}$. The core C3MH-Y module has 19 items, scored on a 5-point Likert scale with total higher scores indicating better engagement in care received in a CAMHS and covers the 3 core domains: management (collaboration); informational (provider knowledge) and relational (interpersonal) continuity. ${ }^{33} 34$ Total scores on all 19 items at the time point preceding discharge from CAMHS will be used as a continuous predictor.

\section{Sample size}

Sample size calculations were conducted for the analytical technique that will be used in the primary research aim, latent growth and growth mixture modelling (GMM). Monte Carlo simulations of latent growth curve models with at least four time points ${ }^{35}$ indicate that a sample size of 250 is sufficient to achieve $80 \%$ power to detect a medium-sized effect of a predictor on the slope growth factor, using four time points. As growth mixture models are more complicated than latent growth modelling, we increased this number by 100 , resulting in a total of 350 .

\section{Data analysis}

Overall, a general latent variable framework will be applied for analyses across aims 1 and 2, starting with the latent growth and GMM to characterise developmental trajectories of the mental health and functioning predictors over the 3-year period. GMM will permit the identification of youth subgroups who follow distinct trajectories of mental health and functioning during the transition period. Separately for each outcome, latent growth models will be specified using a maximum likelihood estimator to determine the overall trajectory. Next, the optimal number of classes will be decided using a range of criteria, including Bayesian Information Criteria, bootstrap likelihood ratio test of $k$ versus $k-1$ groups and the entropy statistic. This analysis will be done using Mplus V.7, ${ }^{36}$ which uses full-information likelihood estimation to account for missing data across assessments. ${ }^{37} 38$

For aim 2, the three-step approach to GMM with covariate $^{35}$ will be used in Mplus 7 to determine predictors of classes of trajectories of mental health and functioning. After deciding on the optimal number of classes (see above), the most likely class variable will be created based on the posterior distribution for each outcome separately. The most likely class then will be regressed on the predictor variables using multinomial logistic regression, considering the misclassification in the second step.

Lastly, for aim 3, the rate of mental health and primary care service use during the pre-CAMHS and post-CAMHS discharge periods will be calculated by dividing the number of visits by the accumulated person months at risk (up to 1-year predischarge and postdischarge). We used the latter specification of time to account for youth having observation periods of different lengths before 
and after discharge (ie, some youth might be discharged before 18 while others may be discharged much later). Using conditional Poisson regression, we will calculate the rate ratio and its 95\% CIs comparing the rates preCAMHS and post-CAMHS discharge periods. ${ }^{39}$ This analysis will be undertaken using $\mathrm{R}^{40}$ For all analyses, two-sided $p<0.05$ will be defined as statistically significant.

\section{Methodological considerations}

Sample attrition (loss to follow-up) is inherent in longitudinal studies; however, recent advances in follow-up management models have demonstrated impressive retention rates $(>90 \%)$ with similar populations. ${ }^{41-43}$ Our retention plan incorporates: rapport building with staff and youth; a dedicated research coordinator; a detailed contact management plan; providing honoraria to participants; and using age-appropriate communication with youth (ie, web, cell phone, text) and other family members including parents and grandparents (with youth consent).

\section{Data management and confidentiality}

All study data will be managed in accordance with the TriAgency principles of digital data management ${ }^{44}$ and the Ontario Personal Health Information Protection Act. Survey data will be entered into Redcap ${ }^{45}$ and downloaded securely into the study database held on a server at the lead study site $(\mathrm{CAMH})$. Participant data will be linked to the health insurance administrative data at the ICES, which houses Ontario's health data. We will transfer the data directly from the CAMH Redcap server to a secure server at ICES via secure data transfer. At ICES, the data will be accessible by a named Data Covenantor. The ICES Covenantor will code the personal health information, replace it with an ICES key number and transfer it to a moated server for the study project.

\section{PATIENT AND PUBLIC INVOLVEMENT}

The LYiTS study is funded by the Canadian Institute for Health Research (CIHR), Canada's federal funding agency for health research. CIHR advocates for patientoriented research ${ }^{46}$ and the inclusion of patients in the research process. As such, LYiTS has included youth and caregivers with lived experience through all phases of the design and management of the study. Partnering with the Margaret and Wallace McCain Centre for Child, Youth \& Family Mental Health Youth Engagement Initiative (YEI) has been instrumental in ensuring that the voice of youth has been incorporated throughout the LYiTS study. ${ }^{47}$ Two youth from the YEI and one caregiver were knowledge user coinvestigators on the grant application, providing feedback on the grant, the research questions, measures and procedures. Youth and caregiver are active participants in the study Expert Advisory Committee (EAC). The EAC and coinvestigators meet regularly to discuss the study protocol and the procedures. Youth have advised on several aspects of LYiTS, including: recruitment strategies (ie, flyers and posters); contact management and retention tools; study measures and instruments; assessment instrument package; and the assessment package's length and readability. Additionally, as part of the EAC and knowledge translation plan, youth and caregivers will be included in the interpretation of findings and their presentation through various knowledge translation activities (eg, presentations and publications).

\section{ETHICS AND DISSEMINATION}

This study protocol has been approved by the REBs at all four recruitment sites. Any protocol modifications will be submitted to corresponding site REBs. Results from this study will be disseminated based on a knowledge mobilisation plan $^{48}$ developed in collaboration with the EAC. These activities include, but are not limited to: (1) peer-reviewed open-access publications; (2) tailored project summaries, developed with input from our knowledge users, created and disseminated through the study website and other mechanisms such as news and social media; (3) workshops and presentations at national and international meetings. Study findings will be reported in accordance with the Strengthening the Reporting of Observational Studies in Epidemiology statement for cohort studies. $^{49}$

\section{Author affiliations}

${ }^{1}$ Lawrence S. Bloomberg Faculty of Nursing, University of Toronto, Toronto, Ontario, Canada

${ }^{2}$ Centre for Addiction and Mental Health, Toronto, Ontario, Canada

${ }^{3}$ Department of Psychiatry, Faculty of Medicine, University of Toronto, Toronto, Ontario, Canada

${ }^{4}$ Health Research Methods, Evidence and Impact (formerly Clinical Epidemiology and Biostatistics), McMaster University Faculty of Health Sciences, Hamilton,

Ontario, Canada

${ }^{5}$ Sunnybrook Health Sciences Centre, Toronto, Ontario, Canada

${ }^{6}$ Department of Psychiatry, Hospital for Sick Children, Toronto, Ontario, Canada ${ }^{7}$ Department of Child Health Evaluative Sciences, Hospital for Sick Children, Toronto, Ontario, Canada

Acknowledgements The authors are very grateful to the Expert Advisory Committee members, including youth from the CAMH Youth Engagement Initiative. They extend their thanks to the CAMHS services and clinicians who are collaborating in this study. KC held the CAMH Chair in Mental Health Nursing Research at the University of Toronto Lawrence S. Bloomberg Faculty of Nursing and the Centre for Addiction and Mental Health during the preparation of this protocol. The authors are grateful to the research assistants, undergraduate and graduate students who are contributing to the operationalisation of the study, particularly Katye Stevens at the Centre for Addiction and Mental Health.

Contributors KC is the principal investigator who conceived the original study design and obtained funding. All authors participated in revisions to the study design for important intellectual content. KC drafted the protocol, and all the authors read, revised and approved the final version of the manuscript. SB is the statistician who led the calculation of the sample size, developed the analytical plan and will undertake the statistical analysis.

Funding The LYiTS project has received funding from the Canadian Institutes of Health Research (CIHR) under grant agreement (PJT-153334). This paper reflects the authors' views. ClHR had no role in the study design, in writing the protocol, or in the decision to submit the protocol for publication.

Competing interests None declared.

Patient consent for publication Not required. 
Provenance and peer review Not commissioned; peer reviewed for ethical and funding approval prior to submission.

Open access This is an open access article distributed in accordance with the Creative Commons Attribution Non Commercial (CC BY-NC 4.0) license, which permits others to distribute, remix, adapt, build upon this work non-commercially, and license their derivative works on different terms, provided the original work is properly cited, appropriate credit is given, any changes made indicated, and the use is non-commercial. See: http://creativecommons.org/licenses/by-nc/4.0/.

\section{ORCID iDs}

Kristin Cleverley http://orcid.org/0000-0002-2822-2129

Joanna Henderson http://orcid.org/0000-0002-9387-5193

\section{REFERENCES}

1 Hofstra MB, Van der Ende J, Verhulst FC. Continuity and change of psychopathology from childhood into adulthood: a 14-year follow-up study. J Am Acad Child Adolesc Psychiatry 2000;39:850-8.

2 Kessler RC, Angermeyer M, Anthony JC, et al. Lifetime prevalence and age-of-onset distributions of mental disorders in the world Health organization's world mental health survey initiative. World Psychiatry 2007;6:168-76.

3 Davidson S, Cappelli M. We've got growing up to do: Policy and practice in youth mental health transitions. Ottawa, Ontario: Canada, 2011. Available: http://www.excellenceforchildandyouth.ca/sites/ default/files/resource/policy_growing_up_to_do.pdf [Accessed 7 Jan 2019].

4 Government of Ontario. Open minds, healthy minds: Ontario's comprehensive mental health and addiction strategy, 2011. Available: http://www.health.gov.on.ca/en/common/ministry/publications/ reports/mental_health2011/mentalhealth_rep2011.pdf

5 Pottick KJ, Bilder S, Vander Stoep A, et al. Us patterns of mental health service utilization for transition-age youth and young adults. $J$ Behav Health Serv Res 2008;35:373-89.

6 Singh SP, Paul M, Ford T, et al. Process, outcome and experience of transition from child to adult mental healthcare: multiperspective study. Br J Psychiatry 2010;197:305-12.

7 Betz CL, O'Kane LS, Nehring WM, et al. Systematic review: Health care transition practice service models. Nurs Outlook 2016;64:229-43.

8 Embrett MG, Randall GE, Longo CJ, et al. Effectiveness of health system services and programs for youth to adult transitions in mental health care: a systematic review of academic literature. Adm Policy Ment Health 2016;43:259-69.

9 Schwartz LA, Brumley LD, Tuchman LK, et al. Stakeholder validation of a model of readiness for transition to adult care. JAMA Pediatr 2013;167:939-46.

10 Coleman EA, Boult C. Improving the quality of transitional care for persons with complex care needs. J Am Geriatr Soc 2003;51:556-7.

11 Blum RWM, Garell D, Hodgman $\mathrm{CH}$, et al. Transition from childcentered to adult health-care systems for adolescents with chronic conditions. Journal of Adolescent Health 1993;14:570-6.

12 Davis $M$. Addressing the needs of youth in transition to adulthood. Adm Policy Ment Health 2003;30:495-509.

13 Health Canada. Reaching for the top: a report by the advisor on healthy children and youth, 2007. Available: http://www.hc-sc.gc.ca/ hl-vs/pubs/child-enfant/advisorconseillere/index-eng.php\#a15

14 McGorry P. Transition to adulthood: the critical period for preemptive, disease-modifying care for schizophrenia and related disorders. Schizophr Bull 2011:37:524-30.

15 Viner R. Transition from paediatric to adult care. bridging the gaps or passing the buck? Arch Dis Child 1999;81:271-5.

16 Paul M, Ford T, Kramer T, et al. Transfers and transitions between child and adult mental health services. Br J Psychiatry.

17 National Institute for Health and Care Excellence. Transition from children's to adults' services for young people using health or social care services: NICE Guideline NG43, 2016. Available: https://www. nice.org.uk/guidance/ng43/resources/transition-from-childrensto-adults-services-for-young-people-using-health-or-social-careservices-pdf-1837451149765 [Accessed 3 Feb 2019].

18 Riosa PB, Preyde M, Porto ML. Transitioning to adult mental health services: perceptions of adolescents with emotional and behavioral problems. Journal of Adolescent Research 2015;30:446-76.

19 Mental Health Commission of Canada. Taking the next step forward: building a responsive mental health and addictions systems for emerging adults. mental health Commission of Canada, 2015. Available: https://www.mentalhealthcommission.ca/sites/
default/files/Taking\%252520the\%252520Next\%252520Step\% 252520Forward_0.pdf [Accessed 3 Feb 2019].

20 Achenbach TM, Rescorla LA. Manual for the ASEBA school-age forms \& profiles. Burlington, VT: University of Vermont, Research Center for Children, Youth, \& Families, 2001.

21 Achenbach TM, Rescorla LA. Manual for the ASEBA adult forms \& profiles. Burlington, VT: University of Vermont Research Center for Children, Youth \& Families, 2003.

22 Goodman A, Goodman R. Strengths and difficulties questionnaire as a dimensional measure of child mental health. J Am Acad Child Adolesc Psychiatry 2009;48:400-3.

23 Goodman R, Meltzer H, Bailey V. The strengths and difficulties questionnaire: a pilot study on the validity of the self-report version. Eur Child Adolesc Psychiatry 1998;7:125-30.

24 Gratz KL, Roemer L. Multidimensional assessment of emotion regulation and dysregulation: development, factor structure, and initial validation of the difficulties in emotion regulation scale. $J$ Psychopathol Behav Assess 2004;26:41-54.

25 Brown RL, Rounds LA. Conjoint screening questionnaires for alcohol and other drug abuse: criterion validity in a primary care practice. Wis Med J 1995;94:135-40.

26 Bird H, Shaffer D, Fisher P, et al. The Columbia-Impairment-Scale (cis): pilot findings on a measure of global impairment for children and adolescents. Int J Methods Psychiatr Res 1993;3:167-76.

27 Wood DL, Sawicki GS, Miller MD, et al. The transition readiness assessment questionnaire (TRAQ): its factor structure, reliability, and validity. Acad Pediatr 2014;14:415-22.

28 Sawicki GS, Lukens-Bull K, Yin X, et al. Measuring the transition readiness of youth with special healthcare needs: validation of the TRAQ - Transition readiness assessment questionnaire. J Pediatr Psychol 2011;36:160-71.

29 Zimet GD, Dahlem NW, Zimet SG, et al. The multidimensional scale of perceived social support. J Pers Assess 1988;52:30-41.

30 Reifman A, Arnett JJ, Colwell MJ. Emerging Adulthood: Theory, Assessment and Application. JYD 2007;2:37-48.

31 O'Mara L, Mueller D, Grypstra L, et al. Does stigma towards menta illness decrease for youth 13-18 years old after participating in school-based youth net Hamilton focus groups? 2009. Available: http://www.excellenceforchildandyouth.ca/sites/default/files/gai attach/rg-127_final_outcomes_report.pdf [Accessed 3 Feb 2019].

32 Singh SP, Paul M, Islam Z, et al. Transition from CAMHS to adult mental health services (track): a study of policies, process and user and carer perspective (track), 2010. Available: http://www.netscc.ac. uk/hsdr/files/project/SDO_FR_08-1613-117 V01.pdf [Accessed 15 May 2017].

33 Tobon JI, Reid GJ, Brown JB. Continuity of Care in Children's Menta Health: Parent, Youth and Provider Perspectives. Community Ment Health J 2015;51:921-30.

34 Haggerty JLet al. Continuity of care: a multidisciplinary review. BMJ 2003;327:1219-21.

35 Asparouhov T, Muthen B. Auxiliary variables in mixture modeling: 3-step approaches using mPLUS. mPLUS web notes: no. 15, 2014. Available: http://www.statmodel.com/download/webnotes/ webnote15.pdf [Accessed 11 Mar 2019]

36 Muthen LK, Muthen BO. Mplus User's Guide. Seventh Edition. Los Angeles, CA, 1998.

37 Little RJA, Rubin DB. Statistical Analysis with Missing Data: Little/ Statistical Analysis with Missing Data. Hoboken, NJ, USA: John Wiley \& Sons, Inc, 2002.

38 Bollen K, Curran P. Latent Curve Models: A Structural Equation Approach. Hoboken: John Wiley \& Sons, 2006.

39 Armstrong BG, Gasparrini A, Tobias A. Conditional Poisson models: a flexible alternative to conditional logistic case cross-over analysis. BMC Med Res Methodol 2014;14:122.

40 Mair P, Wilcox R. Robust statistical methods in R using the WRS2 package. Behav Res Methods 2019;10.

41 Meyers Ket al. What does it take to retain substance-abusing adolescents in research protocols? delineation of effort required, strategies undertaken, costs incurred, and 6-month posttreatment differences by retention difficulty. Drug Alcohol Depend 2003;69:73-85

42 Boys A, Marsden J, Stillwell G, et al. Minimizing respondent attrition in longitudinal research: practical implications from a cohort study of adolescent drinking. J Adolesc 2003;26:363-73.

43 Scott CK. A replicable model for achieving over $90 \%$ follow-up rates in longitudinal studies of substance abusers. Drug Alcohol Depend 2004;74:21-36.

44 Government of Canada. Tri-Agency statement of principles on digital data management. research data management, 2016. Available: http://www.science.gc.ca/eic/site/063.nsf/eng/h_83F7624E.html? OpenDocument [Accessed 3 Feb 2019]. 
45 Harris PA, Taylor R, Thielke R, et al. Research electronic data capture (REDCap) - A metadata-driven methodology and workflow process for providing translational research informatics support. J Biomed Inform 2009;42:377-81.

46 Canadian Institutes of Health Research, Strategy for Patient-Oriented Research - Patient Engagement Framework, 2014. Available: http:// www.cihr-irsc.gc.ca/e/documents/spor framework-en.pdf

47 Heffernan OS, Herzog TM, Schiralli JE, et al. Implementation of a youth-adult partnership model in youth mental health systems research: challenges and successes. Health Expect 2017;20:1183-8.
48 Canadian Institutes of Health Research. Guide to Knowledge Translation Planning at CIHR Integrated and End-of-Grant Approaches. Ottawa: : Canadian Institutes of Health Research, 2012. Available: http://www.cihr-irsc.gc.ca/e/documents/kt_Im_ktplan-en. pdf [Accessed 3 Feb 2019].

49 von EE, Altman DG, Egger M, et al. Strengthening the reporting of observational studies in epidemiology (STROBE) statement: guidelines for reporting observational studies. BMJ 2007;335:806-8. 